\title{
A Log-Derivative Formulation of the Prefactor for the Semiclassical Herman-Kluk Propagator $^{\dagger}$
}

\author{
Ricard Gelabert, Xavier Giménez; Michael Thoss, Haobin Wang, and William H. Miller* \\ Department of Chemistry, University of California-Berkeley, Berkeley, California 94720
}

Received: March 31, 2000; In Final Form: June 26, 2000

\begin{abstract}
A log-derivative formulation of the prefactor term appearing in the semiclassical Herman-Kluk propagator is presented. The resulting new expression is found in practice to avoid the branch cut problem which has hampered previous formulations. The enhanced performance of the log-derivative version of the prefactor has been confirmed by testing it on several one- and two-dimensional model problems. This log-derivative algorithm is also incorporated in the forward-backward initial value representation and applied to a model of the double-slit diffraction problem.
\end{abstract}

\section{Introduction}

Significant effort has been devoted to the development of new methods for describing quantum effects in the dynamics of molecular systems. ${ }^{1}$ Among them, the semiclassical (SC) approach has undergone a rebirth of interest. ${ }^{2}$ In particular, several methods based on initial value representations (IVR) have been proposed ${ }^{3-14}$ and shown capable of accurately describing the dynamics of complex molecular systems, including the description of essentially all types of quantum effects, while being free of the well-known root-search and divergence at caustics problems. This formulation has given rise to a wealth of recent applications which focus, for instance, on model bound state problems, photodissociation, resonance lifetimes and scattering matrix elements, cumulative reaction probabilities and Franck-Condon intensities, and the study of hierarchical spectra. $^{3-14}$

In complex molecular systems, i.e., those with many degrees of freedom, the dynamical quantity of interest is typically expressed in terms of a time correlation function. For such quantities, the forward-backward (FB) approach described by Sun and Miller, ${ }^{1,9 i}$ based on Makri and Thompson's ${ }^{15}$ approach for dealing with influence functionals, is particularly advantageous since it expresses the SC time correlation function as a single phase space average over the initial conditions of trajectories (rather than a double phase space average that would otherwise be required). It seems to be the simplest of SC-IVR approaches that is still capable of describing true quantum coherence effects, and several recent applications to molecular energy transfer, ${ }^{9 \mathrm{j}}$ thermal rate constants, ${ }^{9 \mathrm{k}}$ and simulation of spectra, ${ }^{9 f, 1}$ have shown it to do a reasonably good job. The FB approach has in fact been so effective in simplifying the phase space average over initial conditions (because it greatly diminishes the oscillatory character of the integrand) that the bottleneck in SC-IVR calculations has now become the evaluation of the "prefactor" in the Herman-Kluk (HK) propagator. The prefactor involves the monodromy matrix (the matrix of derivatives of final coordinates and momenta with respect to their initial values), and two aspects of its calculation present difficulties: first, it requires the solution of matrix

\footnotetext{
† Part of the special issue "C. Bradley Moore Festschrift".

$\doteqdot$ On sabbatical leave from the Departament de Química Física, Universitat de Barcelona, Martí i Franquès, 1. 08028 Barcelona, Spain.
}

coupled differential equations, and second, it is given by the square root of a complex quantity, the absolute phase of which must therefore be followed along the trajectory (in order to obtain the correct branch of the complex square root).

The present work proposes an alternate formulation of the prefactor appearing in the SC-IVR-HK approach which has advantages with regard to both of these difficulties. Specifically, we utilize the log-derivative transform ${ }^{18 a}$ to integrate the equations for the monodromy matrix. The log-derivative is typically more slowly varying than the monodromy matrix itself and thus easier to integrate, and the resulting expression for the prefactor also involves the square root in a simpler fashion.

The new formulation for the prefactor is tested herein on a series of model potentials, having a 2-fold purpose in mind. First, the branch cut avoidance has been comprehensively checked, and some formal arguments supporting it are given. Second, the numerical and computational performance of the new algorithm has been established. Finally, a complete semiclassical forward-backward calculation has been carried out to show its actual potentiality. The system chosen has been a model for the double-slit diffraction problem. It is provided here, in addition, as a preliminary check of the semiclassical methodology on a difficult case, one involving pronounced quantum interference effects.

The remainder of the paper is organized as follows. Section 2 deals with the theoretical background. In particular, it includes the general log-derivative formulation of the prefactor, as well as the specific continuity conditions required for the FB approximation. Section 3 shows the application to several model systems, and discusses the results. Finally, section 4 concludes.

\section{Theory}

We start with the expression for the Herman-Kluk, or coherent state version, of the SC-IVR approximation for the quantum mechanical time evolution operator ${ }^{3}$

$$
\begin{aligned}
& \mathrm{e}^{-i \hat{H} t / \hbar}= \\
& \quad(2 \pi \hbar)^{-F} \int \mathrm{d} \mathbf{p}_{0} \int \mathrm{d} \mathbf{q}_{0} C_{t}\left(\mathbf{p}_{0}, \mathbf{q}_{0}\right) \mathrm{e}^{i S_{t}\left(\mathbf{p}_{0}, \mathbf{q}_{0}\right) / \hbar}\left|\mathbf{p}_{t} \mathbf{q}_{t}\right\rangle\left\langle\mathbf{p}_{0} \mathbf{q}_{0}\right|
\end{aligned}
$$

where $F$ is the number of degrees of freedom, $\left(\mathbf{p}_{0}, \mathbf{q}_{0}\right)$ are the initial momenta and coordinates for classical trajectories, $\left(\mathbf{p}_{t}, \mathbf{q}_{t}\right)$ are the classical time-evolved phase space variables, $S_{t}\left(\mathbf{p}_{0}, \mathbf{q}_{0}\right)$ 
is the classical action integral along the trajectory, and $C_{t}$ is the Herman-Kluk prefactor

$$
C_{t}^{2}=2^{-F} \operatorname{det}\left[\mathbf{M}_{q q}+\gamma^{-1} \mathbf{M}_{p p} \gamma+\frac{i}{\hbar} \gamma^{-1} \mathbf{M}_{p q}+\frac{\hbar}{i} \mathbf{M}_{q p} \gamma\right]
$$

where

$$
\begin{aligned}
& \mathbf{M}_{q q}=\frac{\partial \mathbf{q}_{t}}{\partial \mathbf{q}_{0}} \quad \mathbf{M}_{q p}=\frac{\partial \mathbf{q}_{t}}{\partial \mathbf{p}_{0}} \\
& \mathbf{M}_{p q}=\frac{\partial \mathbf{p}_{t}}{\partial \mathbf{q}_{0}} \quad \mathbf{M}_{p p}=\frac{\partial \mathbf{p}_{t}}{\partial \mathbf{p}_{0}}
\end{aligned}
$$

are the elements of the monodromy matrix. According to their definition, each $M$-block is an $F \times F$ matrix, while the global $M$-matrix is $2 F \times 2 F$. In practice, the monodromy matrix is not calculated by performing the derivatives in eq 3 explicitly, but rather by integrating auxiliary equations simultaneously with the classical trajectory itself. For a Cartesian Hamiltonian in mass-weighted coordinates,

$$
H=\sum_{i}^{1} \frac{1}{2} p_{i}^{2}+V(\mathbf{q})
$$

for which the trajectory equations are

$$
\begin{gathered}
\dot{\mathbf{q}}_{t}=\mathbf{p}_{t} \\
\dot{\mathbf{p}}_{t}=-\frac{\partial V}{\partial \mathbf{q}_{t}}
\end{gathered}
$$

these auxiliary equations are

$$
\frac{d}{d t}\left(\begin{array}{ll}
\mathbf{M}_{q q} & \mathbf{M}_{q p} \\
\mathbf{M}_{p q} & \mathbf{M}_{p p}
\end{array}\right)=\left(\begin{array}{ll}
\mathbf{0} & \mathbf{1} \\
-\kappa_{t} & \mathbf{0}
\end{array}\right)\left(\begin{array}{ll}
\mathbf{M}_{q q} & \mathbf{M}_{q p} \\
\mathbf{M}_{p q} & \mathbf{M}_{p p}
\end{array}\right)
$$

where $\kappa_{t}=\partial^{2} V / \partial \mathbf{q} \partial \mathbf{q}$.

As the dimensionality of the problem increases, the bottleneck in a SC-IVR calculation is the prefactor $C_{t}$. The calculation overload originates in the square root from eq 2 , since it is performed on a complex quantity. This operation, as it is wellknown, is not unique, and a branch cut problem appears each time the value of the determinant inside the square root crosses the negative real axis. As a consequence, one usually proceeds in practice keeping track of the kernel of the square root along the trajectory and counting the number of times it crosses the negative real axis. This feature is usually reflected in the theory in the form of the Maslov index. ${ }^{16}$

Keeping track of the square root kernel means calculating a determinant, as shown in eq 2 , for each integration step. It is a complex $F \times F$ determinant, which has to be computed at every time step, small enough to ensure convergence along each trajectory. This determinant evaluation represents a significant part of the SC-IVR calculation. To proceed with the present development, we note that the prefactor in eq 3 can be written as

$$
C_{t}^{2}=\operatorname{det}\left[\frac{1}{2} \mathbf{Q}_{t}+\frac{i}{\hbar} \gamma^{-1} \dot{\mathbf{Q}}_{t}\right]
$$

where the new matrix $\mathbf{Q}$ is defined by

$$
\mathbf{Q}=\mathbf{M}_{q q}+\frac{\hbar}{i} \mathbf{M}_{q p} \gamma
$$

Furthermore, it is not hard to show from eq 6 that $\mathbf{Q}_{t}$ satisfies the following linear second-order differential equation

$$
\ddot{\mathbf{Q}}_{t}+\kappa_{t} \mathbf{Q}_{t}=0
$$

which we observe is equivalent in form to the coupled-channel Schrödinger equation (with $t$ being the "scattering coordinate" and $F$ the number of channels). Much experience in molecular scattering applications over the last three decades has shown that one of the most effective ways of integrating such equations is the log-derivative algorithm (and its close relative, the renormalized Numerov algorithm). The log-derivative matrix $\mathbf{R}_{t}$ is defined by

$$
\mathbf{R}_{t}=\dot{\mathbf{Q}}_{t} \mathbf{Q}_{t}^{-1}
$$

and it is straightforward to show that eq 9 implies the following equation for $\mathbf{R}_{t}$,

$$
\dot{\mathbf{R}}_{t}=-\kappa_{t}-\mathbf{R}_{t}^{2}
$$

with the initial condition

$$
\mathbf{R}_{0}=\frac{\hbar}{i} \gamma
$$

Using the definition of the log-derivative, eq 10, eq 7 for the prefactor can be factored as

$$
C_{t}^{2}=\operatorname{det}\left[\frac{1}{2}\left(\mathbf{1}+\frac{i}{\hbar} \gamma^{-1} \mathbf{R}_{t}\right)\right] \operatorname{det}\left[\mathbf{Q}_{t}\right]
$$

but it is still necessary to express $\operatorname{det}\left(\mathbf{Q}_{t}\right)$ in terms of the $\log$ derivative. This turns out to be quite fascile by noting that

$$
\mathbf{Q}_{t}=T \exp \left[\int_{0}^{t} \mathrm{~d} t^{\prime} \mathbf{R}_{t^{\prime}}\right]
$$

where $T$ is the time-ordering operator. It then follows (see also ref 19) that

$$
\operatorname{det}\left[\mathbf{Q}_{t}\right]=\exp \left[\int_{0}^{t} \mathrm{~d} t^{\prime} \operatorname{Tr}\left(\mathbf{R}_{t^{\prime}}\right)\right]
$$

so that the final (exact) expression for the prefactor in terms of the log-derivative is

$$
C_{t}=\sqrt{\operatorname{det}\left[\frac{1}{2}\left(\mathbf{1}+\frac{i}{\hbar} \gamma^{-1} \mathbf{R}_{t}\right)\right]} \exp \left[\frac{1}{2} \int_{0}^{t} \mathrm{~d} t^{\prime} \operatorname{Tr}\left(\mathbf{R}_{t^{\prime}}\right)\right]
$$

Equation 15, together with eq 11 for the log-derivative, is the primary formal result of the present development.

It is important to note that it has been demonstrated that the determinant of a matrix of the form of $\mathbf{Q}$ is never zero, ${ }^{5 a}$ so that a proper numerical behavior is expected for the $\mathbf{R}$-matrix. Now we turn our attention to the branch cut problem. A branch cut discontinuity appears in eq 7 whenever its imaginary part goes to zero while the real part is negative. In one dimension, one readily shows that, from eq 7 , this case implies

$$
\begin{gathered}
\frac{\partial q_{t}}{\partial q_{0}}+\frac{\partial p_{t}}{\partial p_{0}}<0 \\
\frac{\partial q_{t}}{\partial p_{0}}=\left(\frac{1}{\hbar \gamma}\right)^{2} \frac{\partial p_{t}}{\partial q_{0}}
\end{gathered}
$$

The branch cut condition for the prefactor, in terms of the logderivative R-matrix, can be obtained from eq 15 taking the real 
and imaginary part of the square root kernel. In one dimension, the branch cut condition becomes

$$
\begin{gathered}
1+\frac{1}{\left(\frac{\partial q_{t}}{\partial q_{0}}\right)^{2}+(\hbar \gamma)^{2}\left(\frac{\partial q_{t}}{\partial p_{0}}\right)^{2}}<0 \\
\frac{\partial p_{t}}{\partial q_{0}} \frac{\partial q_{t}}{\partial q_{0}}=-(\hbar \gamma)^{2} \frac{\partial p_{t} \partial q_{t}}{\partial q_{0} \partial p_{0}}
\end{gathered}
$$

Condition 17a never holds in the one-dimensional case because the real part is always positive, and therefore, no branch cut occurs. In the multidimensional case, the real part can have a negative value. However, as it will be shown below, none of the tests performed here led to a simultaneous occurrence of a negative real part and null imaginary part. Thus, for practical purposes, the branch cut problem appears to be avoided.

The form of eq 15 also suggests an approximate treatment that eliminates the square root entirely. It can be easily shown that if we assume that the force constant matrix $\kappa_{t}$ is slowly varying, i.e.,

$$
\frac{\mathrm{d}}{\mathrm{d} t} \frac{\partial^{2} V}{\partial q^{2}}=\frac{\partial^{3} V}{\partial q^{3}} \dot{q} \approx 0
$$

then eq 15 reduces to

$$
C_{t}=\exp \left[\frac{1}{2} \int_{0}^{t} \mathrm{~d} t^{\prime} \operatorname{Tr}\left(\tilde{\mathbf{R}}_{t^{\prime}}\right)\right]
$$

where $\tilde{\mathbf{R}}_{t}$ is a complex matrix that obeys the same differential equation as the $\mathbf{R}_{t}$ matrix, but with the initial condition

$$
\tilde{\mathbf{R}}_{0}=-\frac{i}{2}\left(\frac{1}{\hbar} \gamma^{-1} \kappa_{0}+\hbar \gamma\right)
$$

It is obvious that this approximation eliminates the need of calculating any determinant. An even simpler (but less accurate) approximation is Johnson's "multichannel WKB" approximation: here one sets $\dot{\mathbf{R}}_{t}=0$ in eq $11 \mathrm{a}$, so that

$$
\mathbf{R}_{t} \approx-i\left(\kappa_{t}\right)^{1 / 2}
$$

thus giving the prefactor as

$$
C_{t} \approx \exp \left[-\frac{i}{\hbar} \int_{0}^{t} \mathrm{~d} t^{\prime} \sum_{j=1}^{F} \frac{1}{2} \hbar \omega_{j}\left(t^{\prime}\right)\right]
$$

where $\left\{\omega_{j}(t)^{2}\right\}$ are the eigenvalues of the force constant matrix $\kappa_{t}$; i.e., in this approximation the prefactor is the phase arising from the local zero-point energy along the trajectory. The accuracy of eqs 19 and 20 will be examined in the next section.

Finally, it is interesting to note that the equations which define the $\log$ derivative matrix $\mathbf{R}_{\mathrm{t}}$, eq 10 , and give its time evolution, eq 11a, are essentially the same as those for the matrix $\mathbf{A}$ in Heller's "thawed" Gaussian wave packet approximation (TGA) ${ }^{20}$ (A is the coefficient matrix of the quadratic part of the Gaussian, which characterizes its spreading in coordinate space); cf. eqs 2.5 and 2.4a of ref 20. The matrixes $\mathbf{R}$ and $\mathbf{A}$ are therefore (apart from constants) the same quantity, but they appear in the TGA and the coherent state IVR expressions for the propagator quite differently. Though it is now well-recognized that the HK-IVR is much more accurate than the earlier TGA, it is interesting to see that the same quantity appears in both.
2.1. The Log-Derivative Prefactor within the FB-IVR. So far, the above derivation for this new way of computing the Herman-Kluk prefactor is general. Thus, it can be applied, in principle, to whichever formulation the prefactor is present in. One specific variant, which is being extensively used in our group, is the forward-backward version of the SC-IVR-HK theory. ${ }^{1,9 i-k}$ The FB methodology avoids the double phase space average required in the $\mathrm{HK}-\mathrm{IVR}$ by using trajectories that go forward and backward in time, and may have an instantaneous momentum and position "jump" at an intermediate point. Full details of this formulation have been given elsewhere. ${ }^{1,9 i, k}$

The relevance to the present case is that one needs to know how to integrate the log-derivative $R$-matrix across the "jump" section of the trajectory, since it is governed by a different kind of dynamics. Thus, our purpose here is to derive the new equations satisfied by the $R$-matrix along the jump. We start by noting that the FB-IVR can be viewed as a SC-IVR for a combination of three time propagators:

$$
\exp \left\{-\frac{i}{\hbar} \hat{H}(-t)\right\} \exp \left\{-\frac{i}{\hbar} \tilde{H} \tau\right\} \exp \left\{-\frac{i}{\hbar} \hat{H} t\right\}
$$

where $\hat{H}$ is the standard Hamiltonian for the system under consideration and $\tilde{H}$ is the jump Hamiltonian. Its original time independence is equivalently transformed to an evolution along a time variable, here denoted by $\tau$, and then setting $\tau$ (initial) $=$ 0 and $\tau$ (final $)=1$. Thus, the instantaneous jump can be interpreted, without loss of generality, as an evolution in time from $\tau=0 \rightarrow 1$. Most of the cases considered so far correspond to

$$
\tilde{H}=-\phi(\mathbf{q})
$$

i.e., a function of coordinates only. In this case, the equations of motion for the coordinates and momenta are

$$
\begin{gathered}
\dot{\mathbf{q}}(\tau)=0 \\
\dot{\mathbf{p}}(\tau)=\frac{\partial \phi\left(\mathbf{q}_{t}\right)}{\partial \mathbf{q}_{t}}
\end{gathered}
$$

and one has the trivial solution

$$
\begin{gathered}
\mathbf{q}(\tau=1)=\mathbf{q}_{t} \\
\mathbf{p}(\tau=1)=\mathbf{p}_{t}+\frac{\partial \phi\left(\mathbf{q}_{t}\right)}{\partial \mathbf{q}_{t}}
\end{gathered}
$$

where $\mathbf{p}_{t}$ and $\mathbf{q}_{t}$ are the position and momenta at the end of the forward trajectory, acting as the initial conditions for the jump trajectory. According to eq 26, the expressions for the monodromy matrix elements for this time increment can be written as

$$
\left(\begin{array}{ll}
\mathbf{M}_{q q} & \mathbf{M}_{q p} \\
\mathbf{M}_{p q} & \mathbf{M}_{p p}
\end{array}\right)=\left(\begin{array}{ll}
\mathbf{1} & \mathbf{0} \\
\frac{\partial^{2} \phi}{\partial \mathbf{q}_{t}^{2}} & \mathbf{1}
\end{array}\right)
$$

which, according to the chain rule, leads to the following relation between the "in" (i.e., before the jump) and "out" (after the jump) monodromy matrix elements

$$
\mathbf{M}^{\text {out }}=\left(\begin{array}{ll}
\mathbf{M}_{q q}^{\text {out }} & \mathbf{M}_{q p}^{\text {out }} \\
\mathbf{M}_{p q}^{\text {out }} & \mathbf{M}_{p p}^{\text {out }}
\end{array}\right)=\left(\begin{array}{ll}
\mathbf{1} & \mathbf{0} \\
\frac{\partial^{2} \phi}{\partial \mathbf{q}_{t}^{2}} & \mathbf{1}
\end{array}\right)\left(\begin{array}{ll}
\mathbf{M}_{q q}^{\text {in }} & \mathbf{M}_{q p}^{\text {in }} \\
\mathbf{M}_{p q}^{\text {in }} & \mathbf{M}_{p p}^{\text {in }}
\end{array}\right)
$$


Equation 28 can be used to derive the corresponding in-out relationship for the $\mathbf{Q}$ and $\dot{\mathbf{Q}}$ matrixes. We first have

$$
\begin{aligned}
\mathbf{Q}^{\text {out }} & =\mathbf{M}_{q q}^{\text {out }}+\mathbf{M}_{q p}^{\text {out }} \frac{\hbar}{i} \gamma \\
& =\mathbf{M}_{q q}^{\text {in }}+\mathbf{M}_{q p}^{\text {in }} \frac{\hbar}{i} \gamma=\mathbf{Q}^{\text {in }}
\end{aligned}
$$

and, after a small amount of algebra,

$$
\begin{aligned}
\dot{\mathbf{Q}}^{\text {out }} & =\mathbf{M}_{p p}^{\text {out }} \frac{\hbar}{i} \gamma+\mathbf{M}_{p q}^{\text {out }} \\
& =\mathbf{M}_{p p}^{\text {in }} \frac{\hbar}{i} \gamma+\mathbf{M}_{p q}^{\text {in }}+\frac{\partial^{2} \phi}{\partial q_{t}^{2}}\left(\mathbf{M}_{q q}^{\text {in }}+\mathbf{M}_{q p}^{\text {in }} \frac{\hbar}{i} \gamma\right) \\
& =\dot{\mathbf{Q}}^{\text {in }}+\frac{\partial^{2} \phi}{\partial \mathbf{q}_{t}^{2}} \mathbf{Q}^{\text {in }}
\end{aligned}
$$

Then, from the definition of the log-derivative $\mathbf{R}$-matrix, the above equations yield

$$
\mathbf{R}^{\text {out }}=\dot{\mathbf{Q}}^{\text {out }}\left(\mathbf{Q}^{\text {out }}\right)^{-1}=\left(\dot{\mathbf{Q}}^{\text {in }}+\frac{\partial^{2} \phi}{\partial \mathbf{q}_{t}^{2}} \mathbf{Q}^{\text {in }}\right)\left(\mathbf{Q}^{\text {in }}\right)^{-1}
$$

so that

$$
\mathbf{R}^{\text {out }}=\mathbf{R}^{\text {in }}+\frac{\partial^{2} \phi}{\partial \mathbf{q}_{t}^{2}}
$$

which is the desired result. This expression directly connects the $\mathbf{R}$-matrix right before the jump segment of the trajectory with the one right after, i.e., the initial $\mathbf{R}$-matrix for the backward part of the trajectory.

From the computational point of view, the above derivation ultimately means that, within the FB method, one proceeds integrating the R-matrix first using eq 11a for the forward trajectory, then switching to eq 32 during the jump segment, and then using eq 11a again for the backward part of the trajectory.

\section{Application, Results, and Discussion}

The procedure set forth in the above section is rigorous, i.e., it yields exactly the same results as the conventional method for computing the prefactor through either the SC-IVR-HK method or its FB approximation. As stated above, the main advantage is that the log-derivative formulation of the HK prefactor practically avoids the branch cut problem in the computation of the square root in eq 15. Therefore, it is no longer necessary to keep track of the Maslov index of the prefactor. This means that computationally there is no need to monitor the prefactor value along the integration of the trajectory to ensure a timely update of the Maslov index. As a consequence, the determinant appearing in eq 15 should be calculated only at the end of the trajectory. Besides, the log-derivative version of the prefactor requires the integration of equations 11 , that is, $2 F^{2}$ ordinary differential equations (ODEs) instead of $4 F^{2}$ ODEs in the conventional formulation.

In this section, the reliability and general performance of the log-derivative formulation of the prefactor is thoroughly tested. First, we show results for a series of one- and two-dimensional cases in order to check the accuracy and performance of this

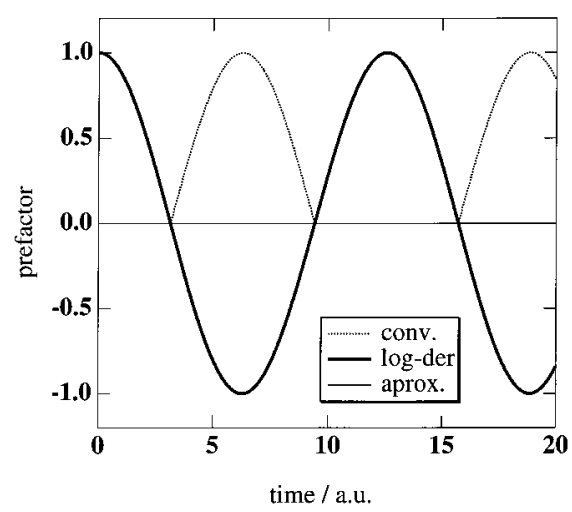

Figure 1. Time dependence of the HK prefactor, for the harmonic potential, from a single trajectory. See text for trajectory's initial conditions. Conv. stands for the conventional formulation, eq 2 ; logder for the logarithmic-derivative version, eq 15; and aprox. for the approximate log-derivative version, eq 19.

approach. The accuracy of the approximate version of it, outlined in section 2, will also be tested. Finally, we will present a complete forward-backward semiclassical calculation on a twodimensional system.

3.1. One-Dimensional Tests. In this section the performance of the log-derivative formulation of the prefactor will be tested on the well-known harmonic oscillator (HO), Morse oscillator (MO), and double-well (DW) problems. Their Hamiltonians are, in mass-weighted coordinates,

$$
\begin{gathered}
\hat{H}_{\mathrm{HO}}=\frac{1}{2} p^{2}+\frac{1}{2} k q^{2} \\
\hat{H}_{\mathrm{MO}}=\frac{1}{2} p^{2}+D\left(\mathrm{e}^{-2 \alpha q}-2 \mathrm{e}^{-\alpha q}\right) \\
\hat{H}_{\mathrm{DW}}=\frac{1}{2} p^{2}+V_{0}-\frac{1}{2} \omega^{2} q^{2}+\frac{\omega^{4} q^{4}}{16 V_{0}}
\end{gathered}
$$

where we have taken $k$ to be unity, $\alpha=0.67 \mathrm{au}^{-1}, \omega$ and $V_{0}$ to be 45000 and $8000 \mathrm{~cm}^{-1}$, respectively, and $D$ equal to 4 au. Figures 1-3 show the real part of the Herman-Kluk prefactor for a single trajectory as a function of time, for each one of the systems, respectively. Initial conditions for the specific trajectories are $q_{0}(\mathrm{HO})=0.95 \mathrm{au}, q_{0}(\mathrm{MO})=-0.35 \mathrm{au}$, and $q_{0}(\mathrm{DW})$ $=-3.0 \mathrm{au}$. In all cases, the initial momentum was set to zero. The initial conditions for the Morse oscillator correspond to a system with energy below the asymptotic limit, whereas the initial conditions for the double well case correspond to a total energy lying above the internal barrier. The prefactor has been computed in three different ways. First, by conventional means, eq 2; second, by exact log-derivative transform, eq 15; and finally, through the approximate log-derivative transformation, eq 19. For the sake of comparison, no tracking of the Maslov index has been done in any case, so as to easily identify the branch cut occurrence and to explicitly show how it is avoided by the log-derivative formulation.

All figures show clearly that the exact log-derivative formulation avoids the branch cuts that affect the conventional formulation, for these one-dimensional cases, as expected from the analysis of the previous section.

Figures 1-3 show also the results corresponding to the approximate log-derivative version. For the harmonic oscillator the approximation is, in fact, exact. The two remaining cases, namely, the Morse and the double-well, consider potentials for which the associated particle dynamics becomes more involved. 


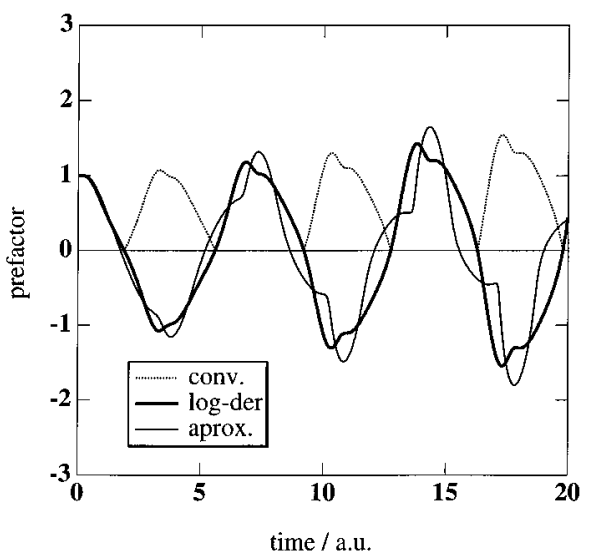

Figure 2. The same as in Figure 1, for a Morse potential.

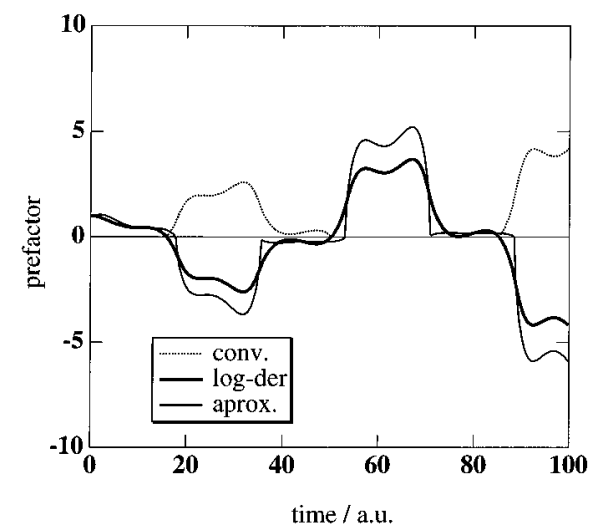

Figure 3. The same as in Figure 1, for a double-well potential.

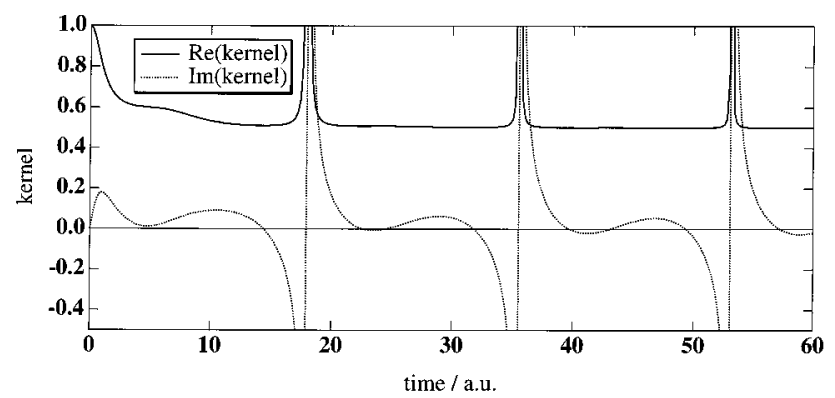

Figure 4. Time dependence of the real and imaginary parts of the kernel of the prefactor's square root, for the double-well potential case.

In particular, it is clearly seen how the prefactor dependence with time shows a more structured behavior and an increasing overall trend. This latter feature is associated to the fact that the trajectory samples regions of configuration space described by potentials having a component of convex curvature. Even with these difficulties, it is seen that the approximate version performs acceptably.

A deeper analysis of the approximate log-derivative version accuracy can be performed from inspection of Figure 4, where the real and imaginary parts of the square root kernel of eq 15 are displayed. The results correspond to a double-well trajectory. It is clearly seen that the approximation to the log-derivative prefactor gives acceptable results, because the log-derivative matrix, in this case, just a scalar, is almost constant except at the points where the trajectory undergoes a classical turning point.

3.2. Two-Dimensional Tests. Next, we proceed to systems of higher dimensionality. The first problem corresponds to a double-well potential in the $y$ direction plus a harmonic oscillator

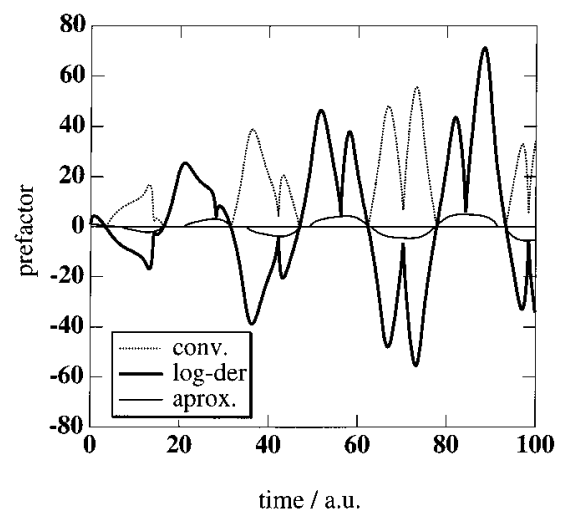

Figure 5. The same as in Figure 1, for the two-dimensional doublewell potential case.

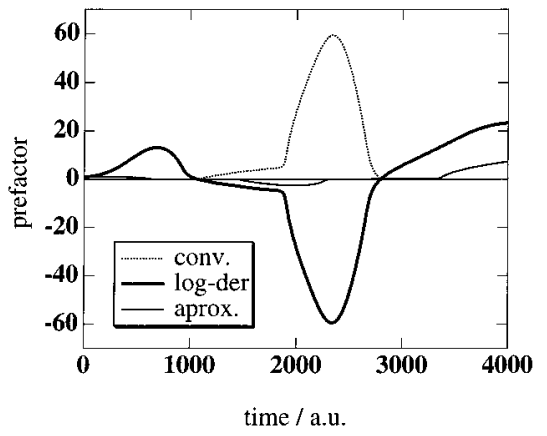

Figure 6. The same as in Figure 1, for the double-slit diffraction experiment model potential case.

in the $x$ direction:

$$
\begin{aligned}
\hat{H}_{\mathrm{DW}}\left(x, y, p_{x}, p_{y}\right)= & \frac{1}{2}\left(p_{x}{ }^{2}+p_{y}{ }^{2}\right)+ \\
& \left(E_{0}-\frac{1}{2} v^{2} y^{2}+\frac{v^{4} y^{4}}{16 E_{0}}\right)+k x^{2}
\end{aligned}
$$

where $E_{0}=8000 \mathrm{~cm}^{-1}, v=42000 \mathrm{~cm}^{-1}$, and $k=0.025 \mathrm{au}$. The second problem is a two-dimensional model for the doubleslit experiment, whereby the double-slit is described as a doublewell potential in the $y$-direction and a Gaussian in the $x$-direction:

$$
\begin{aligned}
\hat{H}_{D . S .}\left(x, y, p_{x}, p_{y}\right)= & \frac{1}{2}\left(p_{x}{ }^{2}+p_{y}{ }^{2}\right)+ \\
& \left(V_{0}-\frac{1}{2} \omega^{2} y^{2}+\frac{\omega^{4} y^{4}}{16 E_{0}}\right) \mathrm{e}^{-(x / \alpha)^{2}}
\end{aligned}
$$

where $V_{0}=8000 \mathrm{~cm}^{-1}, \omega=600 \mathrm{~cm}^{-1}$, and $\alpha=50$ au. Figures 5 and 6 show the real parts of the prefactor for a single trajectory, obtained through the three procedures outlined above. It is again seen that the exact log-derivative formulation avoids the branch cuts while being perfectly accurate, as expected.

The conditions for the branch cut occurrence have been investigated in more detail, for the double slit case, as it is shown in Figure 7. Plotting the real and imaginary parts of the squareroot kernel, as a function of time, shows that the real part becomes negative in the instances where a caustic takes place, as it displays a resonant-like behavior. At these points it is also found that the imaginary part crosses the time axis, thus becoming zero, while describing also a resonant-like pattern. This may indicate a branch cut case. However, a closer inspection reveals that when the imaginary part is zero, the real part is always positive. This has ever been the case for up to 20000 different trajectories individually checked. This example 


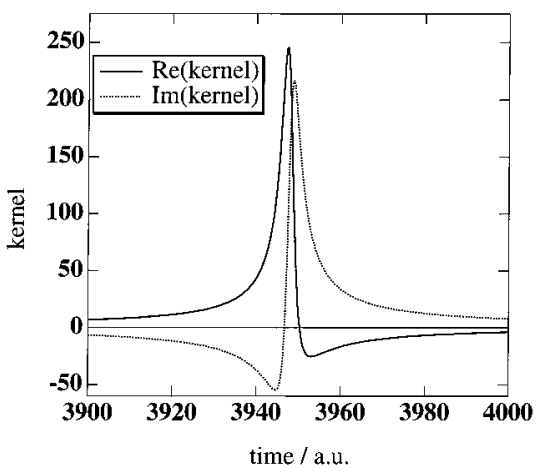

Figure 7. The same as in Figure 4, for the double-slit potential case.

provides, while lacking a rigorous derivation of the branch cut avoidance for multidimensional cases, a confident test on its practical verification.

For the approximate log-derivative version it is found that, contrary to the one-dimensional cases, it is only capable of reproducing the qualitative behavior, being very poor at the quantitative level. Hence, it must be concluded that this approximation can only work for one-dimensional cases.

3.3. Computational Performance of the Log-Derivative in the FB-IVR. The above analysis leads to the conclusion that the integration of the log-derivative equation for the prefactor avoids the branch cut problem, making the calculation of the Maslov index unnecessary. It is still necessary, however, to perform a comprehensive test in order to check the actual performance of the new algorithm.

To that end, this section presents a full FB-SC-IVR calculation of the model system of the double slit, which has been carried out by means of the log-derivative transform of the prefactor. This calculation is relevant since it explicitly tests the continuity of the prefactor along the momentum jump, eq 32 .

The quantity to be calculated, in the double-slit numeric problem, is the angular probability distribution after the particle has gone through the slit, denoted by $P(\vartheta)$. It is given by the following expression:

$$
P(\vartheta)=\left\langle\Psi_{t}|\delta(\vartheta-\hat{\vartheta})| \Psi_{t}\right\rangle=\left\langle\Psi_{i}\left|\mathrm{e}^{i / \hbar \hat{H} t} \delta(\vartheta-\hat{\vartheta}) \mathrm{e}^{-i / \hbar \hat{H} t}\right| \Psi_{i}\right\rangle
$$

where the initial state $\psi_{i}$ is a coherent state (minimum uncertainty) wave packet. The Dirac delta function in eq 35 can be represented by its Fourier series expansion:

$$
P(\vartheta)=\frac{1}{2 \pi} \sum_{l=-\infty}^{+\infty} \mathrm{e}^{-i l \vartheta}\left\langle\psi_{i}\left|\mathrm{e}^{i / \hbar \hat{H} t} \mathrm{e}^{-i l \hat{\vartheta}} \mathrm{e}^{-i / \hbar \hat{H} t}\right| \psi_{i}\right\rangle
$$

so that now the angular probability distribution expression is ready for the FB approximation. Within the HK-IVR it yields

$$
\begin{array}{r}
P(\theta)=\frac{1}{2 \pi_{l}} \sum_{l=-\infty}^{+\infty} \mathrm{e}^{-i l \theta} \frac{1}{(2 \pi \hbar)^{F}} \iint d \mathbf{q}_{0} d \mathbf{p}_{0}\left\langle\mathbf{p}_{0} \mathbf{q}_{0} \mid \psi_{i}\right\rangle \times \\
\left\langle\psi_{i} \mid \mathbf{p}_{0}{ }^{\prime} \mathbf{q}_{0}{ }^{\prime}\right\rangle C_{t}\left(\mathbf{p}_{0}, \mathbf{q}_{0} ; l\right) \mathrm{e}^{i / \hbar S\left(\mathbf{p}_{0}, \mathbf{q}_{0} ; l\right)}
\end{array}
$$

The FB trajectory implicit in eq 37 begins, as usual, with initial conditions $\left(\mathbf{p}_{0} \mathbf{q}_{0}\right)$ at $t=0$ and evolves to time $t$, yielding the position and momenta $\left(\mathbf{p}_{t} \mathbf{q}_{t}\right)$. Then the trajectory undergoes the following momentum jump

$$
\left(\begin{array}{l}
p_{x} \\
\mathrm{p}_{\mathrm{y}}
\end{array}\right)_{t^{\prime}}=\left(\begin{array}{l}
\mathrm{p}_{\mathrm{x}} \\
\mathrm{p}_{\mathrm{y}}
\end{array}\right)_{t}+\frac{l}{\left(x^{2}+y^{2}\right)}\left(\begin{array}{l}
-p_{y} \\
p_{x}
\end{array}\right)_{t}
$$

where, according to the general expressions $24-26$, the jump Hamiltonian is taken to be

$$
\tilde{H}=\phi(\mathbf{q})=l \vartheta=l \arctan \left(\frac{y}{x}\right)
$$

Finally, the trajectory is integrated backward in time, starting at $\left(\mathbf{p}_{t}^{\prime} \mathbf{q}_{t}^{\prime}\right)$ and ending at $t=0$ with position and momenta $\left(\mathbf{p}_{0}^{\prime} \mathbf{q}_{0}^{\prime}\right)$. The overall FB action in eq 37 takes the explicit form:

$$
S\left(\mathbf{p}_{0} \mathbf{q}_{0} ; l\right)=\int_{0}^{t} \mathrm{~d} t^{\prime}[\mathbf{p} \dot{\mathbf{q}}-H]+l \arctan \left(\frac{y}{x}\right)+\int_{t}^{0} \mathrm{~d} t^{\prime}[\mathbf{p} \dot{\mathbf{q}}-H]
$$

The final quantity which has to be calculated explicitly is the log-derivative $R$-matrix time dependence along the momentum jump segment of the trajectory. It is given, according to eq 32 , by

$$
R_{t}^{\prime}=R_{t}+\frac{\partial^{2} \phi}{\partial q_{t}^{2}}=R_{t}+\frac{\partial^{2}}{\partial q_{t}^{2}}\left(l \arctan \frac{y}{x}\right) \quad q_{t}=x_{t}, y_{t}
$$

so that the particularization of the SC-IVR-HK treatment, within the FB approximation and the log-derivative formulation of the prefactor, is completed.

All trajectories were integrated up to 7500 au forward in time using a variable-step Adams method, ${ }^{17}$ and the momentum jump considered values of $l$ up to 50, this being the range of nonnegligible contribution to the Fourier series sum in eq 37. These results were also obtained through use of the conventional formulation of the prefactor, so that the validity of the FBIVR expressions and the general avoidance of the branch cut problem has been checked. A set of 50000 trajectories showed a perfect agreement between both methods, as well as the fact that no branch cut discontinuities during the momentum jump section of the trajectory were observed.

This application provides a good test of the computational performance of the log-derivative algorithm. A detailed analysis of the operations involved, in both the log-derivative and the conventional algorithms, leads to the following considerations. The log-derivative approach requires, as stated, the integration of $2 F^{2}$ differential equations, instead of $4 F^{2}$ for the conventional method. A complex $F \times F$ matrix product is required in eq 11a, corresponding to two $F \times F$ matrix products in eq 6 . Furthermore, the determinant calculation, which in the conventional formulation must be performed at time steps small enough to ensure the proper tracking of the Maslov index, is avoided. Finally, the log-derivative function should be simpler to integrate than the conventional monodromy matrix or the Q-matrix itself. However, this is only true if a specifically taylored algorithm (e.g. the invariant embedding method ${ }^{18}$ ) is used; when standard integrators are used, some difficulties in crossing the resonantlike behavior at caustics may be encountered. Nevertheless, out of this region the log-derivative should behave better and thus be more easily integrated.

The overall results for the present application, concerning the computational performance, show that the CPU time ratios between the two formulations, range between 1.2 and 2.0 in favor of the log-derivative approach, for low (the standard twodimensional double-slit problem) and high dimensionality (five harmonic modes coupled to the double slit) tests, respectively. These results were obtained using a standard integration 


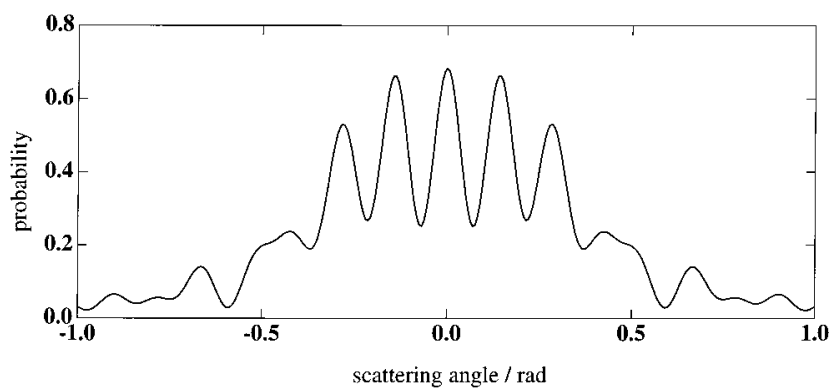

Figure 8. Scattering probability as a function of the double-slit scattering angle.

algorithm in both cases, implying that the integration routines were called about twice as often for the conventional method as for the log-derivative case. These CPU comparisons are not particularly significant, however, for the log-derivative approach can be significantly enhanced by using the invariant embedding algorithm of Johnson and Manolopoulos. Very recently, in fact, we have successfully implemented this algorithm for integrating the log-derivative equation and have obtained very encouraging results. A full account of this new implementation will be given elsewhere. ${ }^{21}$

Finally, the interference pattern obtained in the present case is shown in Figure 8. Even though it is not the main purpose of the present work, it is worth mentioning that the FB approach is able to reproduce, quite accurately, interference phenomena, such as that coming from the double-slit diffraction problem. A comprehensive analysis of this model problem will be reported elsewhere soon. ${ }^{22}$

\section{Conclusions.}

In this paper we have presented a re-formulation of the Herman-Kluk prefactor for the semiclassical time-propagator, based on a log-derivative transform of a combination of monodromy matrix elements. The necessary formulas for applying this new formulation to the forward-backward semiclassical IVR have been worked out. While being a rigorous way of reformulating the prefactor, it avoids in a practical way the branch cut problem that hampers the conventional formulation of the prefactor and thus eliminating the need to monitorize the Maslov index. Thus, it is a new formulation from which further developments or approximations can be derived, so as to tackle the unfavorable scaling of the Herman-Kluk prefactor calculation with the number of degrees of freedom, provided that they preserve the important feature of the branch cut avoidance.

Both the branch cut avoidance and the computational performance have been confirmed for one- and two-dimensional model problems. An approximate version of the log-derivative formulation has also been derived and checked. It has been shown to work reasonably well only for one-dimensional cases. The log-derivative formulation of the SC-IVR propagator has also been applied to a two-dimensional double-slit diffraction problem and shown to yield accurate results of quantum interference.

Acknowledgment. This work was supported by the Director, Office of Science, Office of Basic Energy Sciences, Chemical
Sciences Division of the U.S. Department of Energy under Contract DE-AC03-76SF00098, and by National Science Foundation Grant CHE 97-32758. R.G. thanks the Fundación Ramón Areces in Spain for a postdoctoral fellowship. X. G. acknowledges the Spanish MEC for a "Formación y Movilidad de Profesorado Universitario" fellowship. M. T. gratefully acknowledges a Feodor-Lynen fellowship of the Alexander von Humboldt Foundation.

\section{References and Notes}

(1) Miller, W. H. Faraday Discuss. 1998, 110, 1.

(2) Miller, W. H. J. Chem. Phys. 1970, 53, 3578.

(3) (a) Herman, M. F.; Kluk, E. Chem. Phys. 1984, 91, 27. (b) Kluk, E.; Herman, M. F.; Davis, H. L. J. Chem. Phys. 1986, 84, 326. (c) Herman, M. F. Chem. Phys. Lett. 1997, 275, 445. (d) Guerin, B. E.; Herman, M. F. Chem. Phys. Lett. 1998, 286, 361.

(4) (a) Heller, E. J. J. Chem. Phys. 1991, 94, 2723. (b) 1991, 95, 9431. (c) Grossman, F.; Heller, E. J. Chem. Phys. Lett. 1995, 241, 45.

(5) (a) Kay, K. G. J. Chem. Phys. 1994, 100, 4377; (b) 1994, 100, 4432; (c) 1994, 101, 2250.

(6) (a) Campolieti, G.; Brumer, P. Phys. Rev. A 1994, 50, 997. (b) Provost, D.; Brumer, P. Phys. Rev. Lett. 1995, 74, 250. (c) Campolieti, G.; Brumer, P. J. Chem. Phys. 1998, 109, 2999.

(7) (a) Garashchuk, S.; Tannor, D. J. Chem. Phys. Lett. 1996, 262, 477. (b) Garaschuk, S.; Grossman, F.; Tannor, D. J. J. Chem. Soc., Faraday Trans. 1997, 93, 781. (c) Garashchuk, S.; Tannor, D. J. J. Chem. Phys. 1998, 109, 3028 .

(8) (a) Walton, A. R.; Manolopoulos, D. E. Mol. Phys. 1996, 87, 961. (b) Chem. Phys. Lett. 1995, 244, 448. (c) Brewer, M. L.; Hulme, J. S.; Manolopoulos, D. E. J. Chem. Phys. 1997, 106, 4832.

(9) (a) Miller, W. H. J. Chem. Phys. 1991, 95, 9428. (b) Spath, B. W.; Miller, W. H.J. Chem. Phys. 1996, 104, 95. (c) Sun, X.; Miller, W. H. J. Chem. Phys. 1997, 106, 916; (d) 1997, 106, 6346; (e) 1998, 108, 8870. (f) Batista, V. S.; Zanni, M. T.; Greenblatt, B. J.; Neumark, D. M.; Miller, W. H. J. Chem. Phys. 1999, 110, 3736. (g) Guallar, V.; Batista, V. S.; Miller, W. H. J. Chem. Phys. 1999, 110, 9922. (h) Skinner, D. E.; Miller, W. H. Chem. Phys. Lett. 1999, 300, 20. (i) Sun, X.; Miller, W. H. J. Chem. Phys. 1999, 110, 6635. (j) Skinner, D. E.; Miller, W. H. J. Chem. Phys. 1999, 111, 10787. (k) Wang, H.; Thoss, M.; Miller, W. H. J. Chem. Phys. 2000, 112, 47. (1) Coronado, E. A.; Batista, V. S.; Miller, W. H. J. Chem. Phys. 2000, 112, 5566. (m) Thoss, M.; Miller, W. H.; Stock, G. J. Chem. Phys. 2000, 112, 10282 .

(10) (a) Shalashilin, D. V.; Jackson, B. Chem. Phys. Lett. 1998, 291, 143. (b) Shalashilin, D. V.; Jackson, B. Chem. Phys. Lett. 2000, 318, 305

(11) (a) Stock, G.; Thoss, M. Phys. Rev. Lett. 1997, 78, 578. (b) Thoss, M.; Stock, G. Phys. Rev. A 1999, 59, 64.

(12) van de Sand, G.; Rost, J.-M. Phys. Rev. Lett. 1999, 83, 524.

(13) Grossmann, F. Phys. Rev. A 1999, 60, 1791.

(14) (a) Herman, M. F.; Coker, D. F. J. Chem. Phys. 1999, 111, 1801. (b) Margulis, C. J.; Horner, D. A.; Bonella, S.; Coker, D. F. J. Phys. Chem. 1999, 103, 9552.

(15) (a) Makri, N.; Thompson, K. Chem. Phys. Lett. 1998, 291, 101. (b) Thompson, K.; Makri, N. Phys. Rev. E 1999, 59, R4729.

(16) (a) Delos, J. B. Adv. Chem. Phys. 1986, 65, 161. (b) Gutzwiller, M. C. Chaos in Classical and Quantum Mechanics, Springer-Verlag: New York, 1990

(17) Zwillinger, D. Handbook of Differential Equations, 3rd ed.; Academic Press: San Diego, 1998.

(18) (a) Johnson, B. R. J. Comput. Phys. 1973, 13, 445. (b) Light, J. C.; Walker, R. B. J. Chem. Phys. 1976, 65, 4272. (c) Stechel, E. B.; Walker, R. B.; Light, J. C. J. Chem. Phys. 1978, 69, 3518. (d) Mrugala, F.; Secrest, D. ibid. 1983, 78, 5954. (e) Manolopoulos, D. E. J. Chem. Phys. 1986, 85, 6425. (f) Huarte-Larrañaga, F.; Giménez, X.; Aguilar, A. J. Chem. Phys. 1998, 109, 5761 .

(19) Gradshteyn, I. S.; Ryzhik, I. M. Table of Integrals, Series, and Products; Academic Press: New York, 1994.

(20) Heller, E. J. J. Chem. Phys. 1976, 65, 4979.

(21) Gelabert, R.; Giménez, X.; Miller, W. H. Work in progress.

(22) Gelabert, R.; Giménez, X.; Thoss, M.; Wang, H.; Miller, W. H. J. Chem. Phys. 2000. Submitted for publication. 